\title{
SOME VERSIONS OF ANDERSON'S AND MAHER'S INEQUALITIES I
}

\author{
SALAH MECHERI
}

Received 11 January 2003

\begin{abstract}
We prove the orthogonality (in the sense of Birkhoff) of the range and the kernel of
\end{abstract} an important class of elementary operators with respect to the Schatten $p$-class.

2000 Mathematics Subject Classification: 47A30, 47B47, 47B10, 47B20.

1. Introduction. Let $H$ be a separable infinite-dimensional complex Hilbert space and let $B(H)$ denote the algebra of all bounded operators on $H$ into itself. Given $A, B \in B(H)$, we define the generalized derivation $\delta_{A, B}: B(H) \mapsto B(H)$ by $\delta_{A, B}(X)=A X-X B$ and the elementary operator derivation $\Delta_{A, B}: B(H) \mapsto B(H)$ by $\Delta_{A, B}(X)=A X B-X$. Denote $\delta_{A, A}=\delta_{A}$ and $\Delta_{A, A}=\Delta_{A}$.

In [1, Theorem 1.7], Anderson shows that if $A$ is normal and commutes with $T$, then, for all $X \in B(H)$,

$$
\left\|T+\delta_{A}(X)\right\| \geq\|T\|
$$

It is shown in [10] that if the pair $(A, B)$ has the Fuglede-Putnam property (in particular, if $A$ and $B$ are normal operators) and $A T=T B$, then, for all $X \in B(H)$,

$$
\left\|T+\delta_{A, B}(X)\right\| \geq\|T\|
$$

Duggal [4] showed that the above inequality (1.2) is also true when $\delta_{A, B}$ is replaced by $\Delta_{A, B}$. The related inequality (1.1) was obtained by the author [11] showing that if the pair $(A, B)$ has the Fuglede-Putnam property $(\mathrm{FP})_{C_{p}}$, then

$$
\left\|T+\delta_{A, B}(X)\right\|_{p} \geq\|T\|_{p}
$$

for all $X \in B(H)$, where $C_{p}$ is the von Neumann-Schatten class, $1 \leq p<\infty$, and $\|\cdot\|_{p}$ is its norm for all $X \in B(H)$ and for all $T \in C_{p} \cap \operatorname{ker} \delta_{A, B}$. In all of the above results, $A$ was not arbitrary. In fact, certain normality-like assumptions have been imposed on $A$. A characterization of $T \in C_{p}$ for $1<p<\infty$, which is orthogonal to $R\left(\delta_{A} \mid C_{p}\right)$ (the range of $\left.\delta_{A} \mid C_{p}\right)$ for a general operator $A$, has 
been carried out by Kittaneh [7], showing that if $T$ has the polar decomposition $T=U|T|$, then

$$
\left\|T+\delta_{A}(X)\right\|_{p} \geq\|T\|_{p}
$$

for all $X \in C_{p}(1<p<\infty)$ if and only if $|T|^{p-1} U^{*} \in \operatorname{ker} \delta_{A}$. By a simple modification in the proof of the above inequality, we can prove that this inequality is also true in the general case, that is, if $T$ has the polar decomposition $T=U|T|$, then $\left\|T+\delta_{A, B}(X)\right\|_{p} \geq\|T\|_{p}$ for all $X \in C_{p}(1<p<\infty)$ if and only if $|T|^{p-1} U^{*} \in \operatorname{ker} \delta_{B, A}$. In Sections $1,2,3$, and 4 , we prove these results in the case where we consider $E_{A, B}$ instead of $\delta_{A, B}$, which leads us to prove that if $T \in C_{p}$ and $\operatorname{ker} E_{A, B} \subseteq \operatorname{ker} E_{A, B}^{*}$, then

$$
\left\|T+E_{A, B}(X)\right\|_{p} \geq\|T\|_{p}
$$

for all $X \in C_{p}(1<p<\infty)$ if and only if $T \in \operatorname{ker} E_{A, B}$. In Sections 5 and 6 , we minimize the map $\left\|S+E_{A, B}(X)\right\|_{p}$ and we classify its critical points.

2. Preliminaries. Let $T \in B(H)$ be compact and let $s_{1}(X) \geq s_{2}(X) \geq \cdots \geq 0$ denote the singular values of $T$, that is, the eigenvalues of $|T|=\left(T^{*} T\right)^{1 / 2}$ arranged in their decreasing order. The operator $T$ is said to belong to the Schatten $p$-class $C_{p}$ if

$$
\|T\|_{p}=\left[\sum_{i=1}^{\infty} s_{j}(T)^{p}\right]^{1 / p}=\left[\operatorname{tr}(T)^{p}\right]^{1 / p}, \quad 1 \leq p<\infty,
$$

where tr denotes the trace functional. Hence, $C_{1}$ is the trace class, $C_{2}$ is the Hilbert-Schmidt class, and $C_{\infty}$ is the class of compact operators with

$$
\|T\|_{\infty}=s_{1}(T)=\sup _{\|f\|=1}\|T f\|
$$

denoting the usual operator norm. For the general theory of the Schatten $p$ classes, the reader is referred to $[8,13]$.

Recall that the norm $\|\cdot\|$ of the $B$-space $V$ is said to be Gateaux differentiable at nonzero elements $x \in V$ if

$$
\lim _{t \rightarrow 0, t \in \mathbb{R}} \frac{\|x+t y\|-\|x\|}{t}=\mathscr{R} D_{x}(y)
$$

for all $y \in V$. Here $\mathbb{R}$ denotes the set of reals, $\mathscr{R}$ denotes the real part, and $D_{x}$ is the unique support functional (in the dual space $V^{*}$ ) such that $\left\|D_{x}\right\|=1$ and $D_{x}(x)=\|x\|$. The Gateaux differentiability of the norm at $x$ implies that $x$ is a smooth point of the sphere of radius $\|x\|$. 
It is well known (see [8] and the references therein) that, for $1<p<\infty, C_{p}$ is a uniformly convex Banach space. Therefore, every nonzero $T \in C_{p}$ is a smooth point and, in this case, the support functional of $T$ is given by

$$
D_{T}(X)=\operatorname{tr}\left[\frac{|T|^{p-1} U X^{*}}{\|T\|_{p}^{p-1}}\right]
$$

for all $X \in C_{p}$, where $T=U|T|$ is the polar decomposition of $T$.

DEFINITION 2.1. Let $E$ be a complex Banach space. We define the orthogonality in $E$. We say that $b \in E$ is orthogonal to $a \in E$ if, for all complex $\lambda$, there holds

$$
\|a+\lambda b\| \geq\|a\|
$$

This definition has a natural geometric interpretation, namely, $b \perp a$ if and only if the complex line $\{a+\lambda b \mid \lambda \in \mathbb{C}\}$ is disjoint with the open ball $K(0,\|a\|)$, that is, if and only if this complex line is a tangent one. Note that if $b$ is orthogonal to $a$, then $a$ needs not be orthogonal to $b$. If $E$ is a Hilbert space, then from (2.5), it follows that $\langle a, b\rangle=0$, that is, orthogonality in the usual sense.

3. Main results. In this section, we characterize $T \in C_{p}$ for $1<p<\infty$, which is orthogonal to $R\left(\Delta_{A, B} \mid C_{p}\right)$ (the range of $\left.\Delta_{A, B} \mid C_{p}\right)$ for a general pair of operators $A, B$.

LEMMA 3.1 [7]. Let $u$ and $v$ be two elements of a Banach space $V$ with norm $\|\cdot\|$. If $u$ is a smooth point, then $D_{u}(v)=0$ if and only if

$$
\|u+z v\| \geq\|u\|
$$

for all $z \in \mathbb{C}$ (the complex numbers).

THEOREM 3.2. Let $A, B \in B(H)$ and $T \in C_{p}(1<p<\infty)$. Then

$$
\left\|T+\Delta_{A, B}(X)\right\|_{p} \geq\|T\|_{p}
$$

for all $X \in B(H)$ with $\Delta_{A, B}(X) \in C_{p}$ if and only if $\operatorname{tr}\left(|T|^{p-1} U^{*} \Delta_{A, B}(X)\right)=0$ for all such $X$.

Proof. The theorem is an immediate consequence of equality (2.4) and Lemma 3.1.

THEOREM 3.3. Let $A, B \in B(H)$ and $T \in C_{p}(1<p<\infty)$. Then

$$
\left\|T+\Delta_{A, B}(X)\right\|_{p} \geq\|T\|_{p}
$$

for all $X \in C_{p}$ if and only if $\tilde{T}=|T|^{p-1} U^{*} \in \operatorname{ker} \Delta_{B, A}$. 
Proof. By virtue of Theorem 3.2, it is sufficient to show that $\operatorname{tr}\left(\tilde{T} \Delta_{A, B}(X)\right)=$ 0 for all $X \in C_{p}$ if and only if $\tilde{T} \in \operatorname{ker} \Delta_{B, A}$.

Choose $X$ to be the rank-one operator $f \otimes g$ for some arbitrary elements $f$ and $g$ in $H$; then $\operatorname{tr}(\tilde{T}(A X B-X))=\operatorname{tr}((B \tilde{T} A-\tilde{T}) X)=0$ implies that $\left\langle\Delta_{B, A}(\tilde{T}) f\right.$, $g\rangle=0 \Leftrightarrow \tilde{T} \in \operatorname{ker} \Delta_{B, A}$. Conversely, assume that $\tilde{T} \in \operatorname{ker} \Delta_{B, A}$, that is, $B \tilde{T} A=\tilde{T}$. Since $\tilde{T} X$ and $\tilde{T} \Delta_{B, A}$ are trace classes for all $X \in C_{p}$, we get

$$
\begin{aligned}
\operatorname{tr}(\tilde{T}(A X B-X)) & =\operatorname{tr}(\tilde{T} A X B-\tilde{T} X) \\
& =\operatorname{tr}(X B \tilde{T} A-X \tilde{T}) \\
& =\operatorname{tr}\left(X \Delta_{B, A}(\tilde{T})\right)=0 .
\end{aligned}
$$

LEMmA 3.4. Let $A, B \in B(H)$ and $S \in B(H)$ such that $\operatorname{ker} \Delta_{A, B} \subseteq \operatorname{ker} \Delta_{A^{*}, B^{*}}$. If $A U|S|^{p-1} B=U|S|^{p-1}$, where $p>1$ and $S=U|S|$ is the polar decomposition of $S$, then $A U|S| B=U|S|$.

Proof. If $T=|S|^{p-1}$, then

$$
A U T B=U T
$$

We prove that

$$
A U T^{n} B=U T^{n}
$$

If $A T B=T=A^{*} T B^{*}$, then $B T^{*} T=B T^{*} A T B=T^{*} T B$, and thus $B|T|=|T| B$ and $B T^{2}=T^{2} B$. Since $B$ commutes with the positive operator $T^{2}$, then $B$ commutes with its square roots, that is,

$$
B T=T B
$$

By (3.5) and (3.7) we obtain (3.6). Let $f(t)$ be the map defined on $\sigma(T) \subset R^{+}$ by

$$
f(t)=t^{1 /(p-1)}, \quad 1<p<\infty .
$$

Since $f$ is the uniform limit of a sequence $\left(P_{i}\right)$ of polynomials without constant term (since $f(0)=0$ ), it follows from (3.3) that $A U P_{i}(T) B=U P_{i}(T)$. Therefore, $A U T^{1 /(p-1)} B=U T^{1 /(p-1)}$.

THEOREM 3.5. Let $A$ and $B$ be operators in $B(H)$ such that $\operatorname{ker} \Delta_{A, B} \subseteq$ $\operatorname{ker} \Delta_{A^{*}, B^{*}}$. Then $T \in \operatorname{ker} \Delta_{A, B} \cap C_{p}$ if and only if

$$
\left\|S+\Delta_{A, B}(X)\right\|_{p} \geq\|S\|_{p}
$$

for all $X \in C_{p}$. 
Proof. If $S \in \operatorname{ker} \Delta_{A, B}$, then, by applying [11, Theorem 3.4], it follows that

$$
\left\|S+\Delta_{A, B}(X)\right\|_{p} \geq\|S\|_{p}
$$

for all $X \in C_{p}$. Conversely, if

$$
\left\|S+\Delta_{A, B}(X)\right\|_{p} \geq\|S\|_{p}
$$

for all $X \in C_{p}$, then, from Theorem 3.3, $A|S|^{p-1} U^{*} B=|S|^{p-1} U^{*}$. Since ker $\Delta_{A, B}$ $\subseteq \operatorname{ker} \Delta_{A^{*}, B^{*}}, A^{*}|S|^{p-1} U^{*} B^{*}=|S|^{p-1} U^{*}$. By taking adjoints, we get $A U|S|^{p-1} B$ $=U|S|^{p-1}$. From Lemma 3.4, it follows that $A U|S| B=U|S|$. That is, $S \in \operatorname{ker} \Delta_{A, B}$.

THEOREM 3.6. Let $A, B \in B(H)$. If

(1) $A, B \in \mathscr{L}(H)$ such that $\|A x\| \geq\|x\| \geq\|B x\|$ for all $x \in \mathscr{H}$,

(2) $A$ is invertible and $B$ is such that $\left\|A^{-1}\right\|\|B\| \leq 1$,

(3) $A=B$ is a cyclic subnormal operator,

then, $T \in \operatorname{ker} \Delta_{A, B} \cap C_{p}$ if and only if

$$
\left\|S+\Delta_{A, B}(X)\right\|_{p} \geq\|S\|_{p}
$$

for all $X \in C_{p}$.

Proof. The result of Tong [14, Lemma 1] guarantees that the above condition implies that for all $T \in \operatorname{ker}\left(\delta_{A, B} \mid \mathscr{K}(\mathscr{H})\right), \overline{R(T)}$ reduces $A, \operatorname{ker}(T)^{\perp}$ reduces $B$, and $\left.A\right|_{\overline{R(T)}}$ and $\left.B\right|_{\operatorname{ker}(T)^{\perp}}$ are unitary operators. Take $\mathscr{H}_{1}=\mathscr{H}=\overline{\operatorname{ran} S} \oplus \overline{\operatorname{ran} S}$ and $\mathscr{H}_{2}=\mathscr{H}=\operatorname{ker} S \oplus \operatorname{ker} S^{\perp}$. According to the decomposition of $\mathscr{H}$ and for $A_{1}: \mathscr{H}_{1} \rightarrow \mathscr{H}_{1}, A_{2}: \mathscr{H}_{2} \rightarrow \mathscr{H}_{2}$, and $S: \mathscr{H}_{2} \rightarrow \mathscr{H}_{1}$, we can write

$$
A=\left(\begin{array}{cc}
A_{1} & 0 \\
0 & A_{2}
\end{array}\right), \quad B^{*}=\left(\begin{array}{cc}
B_{1}^{*} & 0 \\
0 & B_{2}^{*}
\end{array}\right), \quad S=\left(\begin{array}{cc}
S_{1} & 0 \\
0 & 0
\end{array}\right) .
$$

From $A S B=S$, it follows that $A_{1} S B_{1}=S$, and since $A_{1}$ and $B_{1}$ are unitary operators, we obtain $A_{1}^{*} S B_{1}^{*}=S$, and the result holds by the above theorem.

The above inequality holds in particular if $A=B$ is isometric; in other words, $\|A x\|=\|x\|$ for all $x \in \mathscr{H}$.

(2) In this case, it suffices to take $A_{1}=\|B\|^{-1} A$ and $B_{1}=\|B\|^{-1} B$, then $\left\|A_{1} x\right\| \geq\|x\| \geq\left\|B_{1} x\right\|$, and the result holds by (1) for all $x \in \mathscr{H}$.

(3) Since $T$ commutes with $A$, it follows that $T$ is subnormal [15]. But any compact subnormal operator is normal; hence, $T$ is normal. By applying Fuglede-Putnam theorem, we get that $A T A=T$ implies $A^{*} T A^{*}=T$.

4. The case where $n>1$. Let $A=\left(A_{1}, A_{2}, \ldots, A_{n}\right)$ and $B=\left(B_{1}, B_{2}, \ldots, B_{n}\right)$ be $n$-tuples of operators in $B(H)$. In this section, we characterize $T \in C_{p}$ for $1<$ $p<\infty$, which is orthogonal to $R\left(E_{A, B} \mid C_{p}\right)$ (the range of $E_{A, B} \mid C_{p}$ ) for a general pair of operators $A$ and $B$. 
By the same argument used in the proofs of Theorems 3.2 and 3.3, we prove the following theorems.

THEOREM 4.1. Let $A=\left(A_{1}, A_{2}, \ldots, A_{n}\right)$ and $B=\left(B_{1}, B_{2}, \ldots, B_{n}\right)$ be $n$-tuples of operators in $B(H)$ and $T \in C_{p}(1<p<\infty)$. Then

$$
\left\|T+E_{A, B}(X)\right\|_{p} \geq\|T\|_{p}
$$

for all $X \in B(H)$ with $E_{A, B}(X) \in C_{p}$ if and only if $\operatorname{tr}\left(|T|^{p-1} U^{*} E_{A, B}(X)\right)=0$ for all such $X$.

THEOREM 4.2. Let $A=\left(A_{1}, A_{2}, \ldots, A_{n}\right)$ and $B=\left(B_{1}, B_{2}, \ldots, B_{n}\right)$ be n-tuples of operators in $B(H)$ and $T \in C_{p}(1<p<\infty)$. Then

$$
\left\|T+E_{A, B}(X)\right\|_{p} \geq\|T\|_{p}
$$

for all $X \in C_{p}$ if and only if $\tilde{T}=|T|^{p-1} U^{*} \in \operatorname{ker} E_{A, B}$.

LEMmA 4.3. Let $C=\left(C_{1}, C_{2}, \ldots, C_{n}\right)$ be $n$-tuple of operators in $B(H)$ such that $\sum_{i=1}^{n} C_{i} C_{i}^{*} \leq 1, \sum_{i=1}^{n} C_{i}^{*} C_{i} \leq 1$, and $\operatorname{ker} E_{C} \subseteq \operatorname{ker} E_{C^{*}}$. If

$$
\sum_{i=1}^{n} C_{i} U|S|^{p-1} C_{i}=U|S|^{p-1},
$$

where $p>1$ and $S=U|S|$ is the polar decomposition of $S$, then

$$
\sum_{i=1}^{n} C_{i} U|S| C_{i}=U|S| .
$$

Proof. If $T=|S|^{p-1}$, then

$$
\sum_{i=1}^{n} C_{i} U T C_{i}=U T
$$

We prove that

$$
\sum_{i=1}^{n} C_{i} U T^{n} C_{i}=U T^{n} .
$$

It is known that if $\sum_{i=1}^{n} C_{i} C_{i}^{*} \leq 1, \sum_{i=1}^{n} C_{i}^{*} C_{i} \leq 1$, and $\operatorname{ker} E_{c} \subseteq \operatorname{ker} E_{c}^{*}$, then the eigenspaces corresponding to distinct nonzero eigenvalues of the compact positive operator $|S|^{2}$ reduce each $C_{i}$ (see [3, Theorem 8], [14, Lemma 2.3]). In particular, $|S|$ commutes with $C_{i}$ for all $1 \leq i \leq n$. This implies also that $|S|^{p-1}=T$ commutes with each $C_{i}$ for all $1 \leq i \leq n$. Hence $C_{i}|T|=|T| C_{i}$ and $C_{i} T^{2}=T^{2} C_{i}$. 
Since $C_{i}$ commutes with the positive operator $T^{2}$, then $C_{i}$ commutes with its square roots, that is,

$$
C_{i} T=T C_{i}
$$

By the same arguments used in the proof of Lemma 3.4, the proof of this lemma can be completed.

THEOREM 4.4. Let $C=\left(C_{1}, C_{2}, \ldots, C_{n}\right)$ be $n$-tuple of operators in $B(H)$ such that $\sum_{i=1}^{n} C_{i} C_{i}^{*} \leq 1, \sum_{i=1}^{n} C_{i}^{*} C_{i} \leq 1$, and $\operatorname{ker} E_{C} \subseteq \operatorname{ker} E_{C^{*}}$. Then $S \in \operatorname{ker} E_{C} \cap C_{p}$ $(1<p<\infty)$ if and only if

$$
\left\|S+E_{C}(X)\right\|_{p} \geq\|S\|_{p}
$$

for all $X \in C_{p}$.

Proof. If $S \in \operatorname{ker} E_{C}$, then, from [14, Theorem 2.4], it follows that $\| S+$ $E_{C}(X)\left\|_{p} \geq\right\| S \|_{p}$ for all $X \in C_{p}$. Conversely, if $\left\|S+E_{C}(X)\right\|_{p} \geq\|S\|_{p}$ for all $X \in C_{p}$, then, from Theorem 4.2, $\sum_{i=1}^{n} C_{i}|S|^{p-1} U^{*} C_{i}=|S|^{p-1} U^{*}$. Since ker $E_{C} \subseteq$ $\operatorname{ker} E_{C^{*}}, \sum_{i=1}^{n} C_{i}^{*}|S|^{p-1} U^{*} C_{i}^{*}=|S|^{p-1} U^{*}$. Taking adjoints, we get $\sum_{i=1}^{n} C_{i} U$ $\times|S|^{p-1} C_{i}=U|S|^{p-1}$, and from Lemma 4.3, it follows that $\sum_{i=1}^{n} C_{i} U|S| C_{i}=$ $U|S|$, that is, $S \in \operatorname{ker} E_{C}$.

THEOREM 4.5. Let $A=\left(A_{1}, A_{2}, \ldots, A_{n}\right)$ and $B=\left(B_{1}, B_{2}, \ldots, B_{n}\right)$ be $n$-tuples of operators in $B(H)$ such that $\sum_{i=1}^{n} A_{i} A_{i}^{*} \leq 1, \sum_{i=1}^{n} A_{i}^{*} A_{i} \leq 1, \sum_{i=1}^{n} B_{i} B_{i}^{*} \leq 1$, $\sum_{i=1}^{n} B_{i}^{*} B_{i} \leq 1$, and $\operatorname{ker} E_{A, B} \subseteq \operatorname{ker} E_{A^{*}, B^{*}}$.

Then $T \in \operatorname{ker} E_{A, B} \cap C_{p}$ if and only if

$$
\left\|S+E_{A, B}(X)\right\|_{p} \geq\|S\|_{p}
$$

for all $X \in C_{p}$.

Proof. It suffices to take the Hilbert space $H \oplus H$ and the operators

$$
C_{i}=\left[\begin{array}{cc}
A_{i} & 0 \\
0 & B_{i}
\end{array}\right], \quad S=\left[\begin{array}{ll}
0 & T \\
0 & 0
\end{array}\right], \quad X=\left[\begin{array}{ll}
0 & X \\
0 & 0
\end{array}\right]
$$

and apply Theorem 4.4 .

5. Remarks. (1) It is known (see [8] and the references therein) that the smooth points of $K(H)$ are those compact operators that attain their norm at a unique (up to multiplication by a constant of modulus one) unit vector. It has been shown in [8] that a nonzero $T \in B(H)$ is a smooth point if and only if $T$ attains its norm at a unique (up to multiplication by a constant of modulus one) unit vector $e \in H$ and $\|T\|_{e} \leq\|T\|$, where $\|T\|_{e}$ is the essential 
norm of $T$, that is, the norm of $\pi(T)$, where $\pi$ is the quotient map of $B(H)$ onto $B(H) / K(H)$. In this case,

$$
D_{T}(X)=\operatorname{tr}\left[\frac{(e \otimes T e)}{\|T\|} X\right]=\left\langle X e, \frac{T e}{\|T\|}\right\rangle
$$

for all $X \in B(H)$, where $\langle\cdot, \cdot\rangle$ denotes the inner product on $H$ and $e \otimes T e$ is the rank-one operators defined by $(e \otimes T e) f=\langle f, T e\rangle e$ for all $f \in H$.

Hence, for the usual operator norm, Theorems 3.2, 3.3, 4.1, and 4.2 can be combined in the following formulation. Let $A, B \in B(H)$ and $T \in B(H)$ be a smooth point. If $\tilde{T}=e \otimes T e$, then the following statements are equivalent:

(i) $\left\|T+E_{A, B}(X)\right\| \geq\|T\|$ for all $X \in B(H)$,

(ii) $\operatorname{tr}\left(T^{\sim} E_{A, B}(X)\right)=0$ for all $X \in B(H)$,

(iii) $\tilde{T} \in \operatorname{ker} E_{A, B}$.

(2) It is still possible to give a characterization similar to this given in the usual operator norm for the norm $\|\cdot\|_{\infty}$. However, in this case, we have to assume that $T$ is a smooth point, that is, the given norm is Gateaux differentiable at $T$ and $\tilde{T}=e \otimes T e$, where $e$ is the unique (up to multiplication by a constant of modulus one) unit vector at which $T$ attains its norm.

(3) It is well known that the Hilbert-Schmidt class $C_{2}$ is a Hilbert space under the inner product $\langle Y, Z\rangle=\operatorname{tr} Z^{*} Y$.

We remark here that, for the Hilbert Schmidt norm $\|\cdot\|_{2}$, the orthogonality results in Theorems 3.3, 3.5, 4.1, and 4.2 are to be understood in the usual Hilbert-space sense. Note in the case $\tilde{T}=|T| U^{*}=T^{*}$ that

$$
\left\|T+E_{A, B}(X)\right\|_{2}^{2}=\left\|E_{A, B}(X)\right\|_{2}^{2}+\|T\|_{2}^{2}
$$

for all $X \in C_{2}$ if and only if $T^{*} \in \operatorname{ker} E_{A, B}$.

(4) Theorem 4.4 does not hold in the case $0<p \leq 1$ because the functional calculus argument involving the function $t \mapsto t^{1 /(p-1)}$, where $0 \leq t<\infty$, is only valid for $1<p<\infty$. We ask if there is another proof where this theorem still holds in the case $0<p<1$. For the case $p=1$, this theorem still holds see [12, Theorem 2.3].

6. On minimizing $\|T-(A X B-X)\|_{p}^{p}$. Maher [9, Theorem 3.2] showed that, if $A$ is normal, $A T=T A, 1 \leq p<\infty$, and $S \in \operatorname{ker} \delta_{A, B} \cap C_{p}$; then the map $F_{p}$ defined by $F_{p}(X)=\|S-(A X-X A)\|_{p}^{p}$ has a global minimizer at $V$ if, and for $1<p<\infty$ only if, $A V-V A=0$. In other words, we have

$$
\|S-(A X-X A)\|_{p}^{p} \geq\|T\|_{p}^{p}
$$

if, and for $1<p<\infty$ only if, $A V-V A=0$. In [10] we generalized Maher's result, showing that if the pair $(A, B)$ has the property $(\mathrm{FP})_{C_{p}}$, that is, $(A T=T B$, where $T \in C_{p}$ implies $\left.A^{*} T=T B^{*}\right), 1 \leq p<\infty$ and $S \in \operatorname{ker} \delta_{A, B} \cap C_{p}$, then the map $F_{p}$ 
defined by $F_{p}(X)=\|S-(A X-X B)\|_{p}^{p}$ has a global minimizer at $V$ if, and for $1<p<\infty$ only if, $A V-V B=0$. In other words, we have

$$
\|S-(A X-X B)\|_{p}^{p} \geq\|T\|_{p}^{p}
$$

if, and for $1<p<\infty$ only if, $A V-V B=0$. In this paper, we obtain an inequality similar to (6.1), where the operator $A X-X B$ is replaced by the operator $\Delta_{A, B}(X)=A X B-X$ (in the case $n=1$ ). We prove that if $\operatorname{ker} \Delta_{A, B} \subseteq \operatorname{ker} \Delta_{A^{*}, B^{*}}$ and $T \in \operatorname{ker} \Delta_{A, B} \cap C_{p}$, then the map $F_{p}$ defined by $F_{p}(X)=\|T-(A X B-X)\|_{p}^{p}$ has a global minimizer at $V$ if, and for $1<p<\infty$ only if, $A V B-V=0$. In other words, we have

$$
\|T-(A X B-X)\|_{p}^{p} \geq\|T\|_{p}^{p}
$$

if, and for $1<p<\infty$ only if, $A V B-V=0$. Additionally, we show that if $\operatorname{ker} \Delta_{A, B} \subseteq \operatorname{ker} \Delta_{A^{*}, B^{*}}$ and $T \in \operatorname{ker} \Delta_{A, B} \cap C_{p}, 1<p<\infty$, then the map $F_{p}$ has a critical point at $W$ if and only if $A W B-W=0$, that is, if $\mathscr{D}_{W} F_{p}$ is the Frechet derivative at $W$ of $F_{p}$, the set

$$
\left\{W \in \mathscr{B}(H): \mathscr{D}_{W} F_{p}=0\right\}
$$

coincides with $\operatorname{ker} \Delta_{A, B}$ (the kernel of $\Delta_{A, B}$ ).

THEOREM 6.1 [2]. If $1<p<\infty$, then the map

$$
F_{p}: C_{p} \longmapsto \mathbb{R}^{+}
$$

defined by $X \mapsto\|X\|_{p}^{p}$ is differentiable at every $X \in C_{p}$ with derivative $\mathscr{D}_{X} F_{p}$ given by

$$
\mathscr{D}_{X} F_{p}(T)=p \operatorname{Retr}\left(|X|^{p-1} U^{*} T\right),
$$

where $\operatorname{tr}$ denotes trace, $\operatorname{Re} z$ is the real part of a complex number $z$, and $X=U|X|$ is the polar decomposition of $X$. If $\operatorname{dim} \mathscr{H}<\infty$, then the same result holds for $0<p \leq 1$ at every invertible $X$.

THEOREM 6.2 [6]. If $u$ is a convex set of $C_{p}$ with $1<p<\infty$, then the map $X \mapsto\|X\|_{p}^{p}$, where $X \in \mathcal{U}$, has at most a global minimizer.

Definition 6.3. Let $\mathcal{U}(A, B)=\left\{X \in B(H): A X B-X \in C_{p}\right\}$ and let $F_{p}: \cup \mapsto$ $\mathbb{R}^{+}$be the map defined by $F_{p}(X)=\|T-(A X B-X)\|_{p}^{p}$, where $T \in \operatorname{ker} \Delta_{A, B} \cap C_{p}$ $(1 \leq p<\infty)$.

By a simple modification in the proof of Lemma 4.3, we can proof the following lemma.

LEMMA 6.4. Let $A, B \in B(H)$ and $S \in B(H)$ such that $\operatorname{ker} \Delta_{A, B} \subseteq \operatorname{ker} \Delta_{A^{*}, B^{*}}$. If $A|S|^{p-1} U^{*} B=|S|^{p-1} U^{*}$, where $p>1$ and $S=U|S|$ is the polar decomposition of $S$, then $A|S| U^{*} B=|S| U^{*}$. 
THEOREM 6.5. Let $A, B \in B(H)$. If $\operatorname{ker} \Delta_{A, B} \subseteq \operatorname{ker} \Delta_{A^{*}, B^{*}}$ and $T \in \operatorname{ker} \Delta_{A, B} \cap$ $C_{p}$, then, for $1 \leq p<\infty$, the map $F_{p}$ has a global minimizer at $W$ if, and for $1<p<\infty$ only if, $A W B-W=0$.

Proof. If ker $\Delta_{A, B} \subseteq \operatorname{ker} \Delta_{A^{*}, B^{*}}$, then it follows from Theorem 3.5 that $\| T-$ $(A X B-X)\left\|_{p}^{p} \geq\right\| T \|_{p}^{p}$, that is, $F_{p}(X) \geq F_{p}(W)$. Conversely, if $F_{p}$ has a minimum, then

$$
\|T-(A W B-W B)\|_{p}^{p}=\|S\|_{p}^{p}
$$

Since $\mathcal{U}$ is convex, then the set $\mathscr{V}=\{T-(A X B-X) ; X \in \mathcal{U}\}$ is also convex. Thus Theorem 6.2 implies that $S-(A W B-W)=S$.

THEOREM 6.6. Let $A, B \in B(H)$. If $\operatorname{ker} \Delta_{A, B} \subseteq \operatorname{ker} \Delta_{A^{*}, B^{*}}$ and $S \in \operatorname{ker} \Delta_{A, B} \cap$ $C_{p}$, then, for $1<p<\infty$, the map $F_{p}$ has a critical point at $W$ if and only if $A W B-W=0$.

Proof. Let $W, S \in \mathcal{U}$ and let $\phi$ and $\varphi$ be two maps defined, respectively, by $\phi: X \mapsto S-(A X B-X)$ and $\varphi: X \mapsto\|X\|_{p}^{p}$.

Since the Frechet derivative of $F_{p}$ is given by

$$
\mathscr{D}_{W} F_{p}(T)=\lim _{h \rightarrow 0} \frac{F_{p}(W+h T)-F_{p}(W)}{h},
$$

it follows that $\mathscr{D}_{W} F_{p}(T)=\left[\mathscr{D}_{S-(A W B-W)}\right](A T B-T)$. If $W$ is a critical point of $F_{p}$, then $\mathscr{D}_{W} F_{p}(T)=0$ for all $T \in \mathscr{U}$. By applying Theorem 6.1, we get

$$
\begin{aligned}
\mathscr{D}_{W} F_{p}(T) & =p \operatorname{Retr}\left[|S-(A W B-W)|^{p-1} W^{*}(A T B-T)\right] \\
& =p \operatorname{Retr}[Y(A T B-T)]=0,
\end{aligned}
$$

where $S-(A W B-W)=W|S-(A W B-W)|$ is the polar decomposition of the operator $S-(A W B-W)$, and $Y=|S-(A W B-W)|^{p-1} W^{*}$.

An easy calculation shows that $A Y B-Y=0$, that is,

$$
A|S-(A W B-W)|^{p-1} W^{*} B=|S-(A W B-W)|^{p-1} W^{*} .
$$

It follows from Lemma 6.4 that

$$
A|S-(A W B-W)| W^{*} B=|S-(A W B-W)| W^{*} .
$$

By taking adjoints and since $\operatorname{ker} \Delta_{A, B} \subseteq \operatorname{ker} \Delta_{A^{*}, B^{*}}$, we get $A(T-(A W B-$ $W)) B=(T-(A W B-W))$. Then $A(A W B-W) B=(A W B-W)$.

Hence $A W B-W \in R\left(\Delta_{A, B}\right) \cap \operatorname{ker} \Delta_{A, B}$. It is easy to see that (arguing as in the proof of Theorem 3.5) if $A, B \in \mathscr{B}(H)$, $\operatorname{ker} \Delta_{A, B} \subseteq \operatorname{ker} \Delta_{A^{*}, B^{*}}$, and $T \in \operatorname{ker} \Delta_{A, B}$, 
where $T \in \mathscr{B}(H)$, then

$$
\|T-(A X B-X)\| \geq\|T\|
$$

holds for all $X \in \mathscr{B}(H)$ and for all $T \in \operatorname{ker} \Delta_{A, B}$. Hence $A W B-W=0$.

Conversely, if $A W B=W$, then $W$ is a minimum, and since $F_{p}$ is differentiable, then $W$ is a critical point.

THEOREM 6.7. Let $A, B \in \mathscr{B}(H)$ such that $\operatorname{ker} \Delta_{A, B} \subseteq \operatorname{ker} \Delta_{A^{*}, B^{*}}, S \in$ $\operatorname{ker} \Delta_{A, B} \cap C_{p}(0<p \leq 1), \operatorname{dim} \mathscr{H}<\infty$, and $S-(A W B-W)$ is invertible. Then $F_{p}$ has a critical point at $W$ if $A W B-W=0$.

Proof. Suppose that $\operatorname{dim} \mathscr{H}<\infty$. If $A W B-W=0$, then $S$ is invertible by hypothesis. Also $|S|$ is invertible, hence $|S|^{p-1}$ exists for $0<p \leq 1$. If we take

$$
Y=|S|^{p-1} U^{*}
$$

with $S=U|S|$ the polar decomposition and since $A S B=S$ implies $B S^{*} A=S^{*}$, then $A S^{*} S=A S^{*} B S A=S^{*} S A$, and this implies that $|S|^{2} A=A|S|^{2}$ and $|S| A=$ $A|S|$.

Since $B S^{*} A=S^{*}$, that is, $A|S| U^{*} B=|S| U^{*},|S|\left(A U^{*} B-U^{*}\right)=0$, and since $A|S|^{p-1}=|S|^{p-1} A$, then

$$
A Y B-Y=A|S|^{p-1} U^{*} B-|S|^{p-1} U^{*}=|S|^{p-1}\left(A U^{*} B-U^{*}\right)
$$

so that $A Y B-Y=0$ and $\operatorname{tr}[(A Y B-Y) T]=0$ for all $T \in B(H)$. Since $S=S-$ $(A W B-W)$, then

$$
\begin{aligned}
0 & =\operatorname{tr}[Y A T B-Y A T]=\operatorname{tr}[Y(A T B-T)] \\
& =p \operatorname{Retr}[Y(A T B-A T)]=p \operatorname{Retr}\left[|S|^{p-1} U^{*}(A T B-T)\right] \\
& =\left(\mathscr{D}_{T} \phi\right)(A T B-T)=\left(\mathscr{D}_{W} F_{p}\right)(T) .
\end{aligned}
$$

REMARK 6.8. (1) In Theorem 6.6 , the implication " $W$ is a critical point $\Rightarrow$ $A W B-W B=0$ " does not hold in the case $0<p \leq 1$ because the functional calculus argument involving the function $t \mapsto t^{1 /(p-1)}$, where $0 \leq t<\infty$, is only valid for $1<p<\infty$.

(2) Theorems 3.5, 6.5, 6.6, and 6.7 hold in particular if $A$ and $B$ are contractions. Indeed, it is known [4] that if $A$ and $B$ are contractions and $\Delta_{A, B}(S)=0$, where $S \in C_{p}$, then $\Delta_{A^{*}, B^{*}}(S)=\delta_{A^{*}, B}(S)=\delta_{A, B^{*}}(S)=0$.

(3) The set

$$
\mathscr{S}=\left\{X: A X B-X \in C_{p}\right\}
$$

contains $C_{p}$ for if $X \in C_{p}$, then $X \in \mathscr{Y}$ and, for example, $I \in \mathscr{Y}$ but $I \notin C_{p}$. If $A \in C_{p}$, the conclusions of Theorems $6.5,6.6$, and 6.7 hold for all $X \in B(H)$. 
7. On minimizing $\left\|T-\left(\sum_{i=1}^{n} A_{i} X B_{i}-X\right)\right\|_{p}^{p}$. Let $A=\left(A_{1}, A_{2}, \ldots, A_{n}\right)$ and $B=\left(B_{1}, B_{2}, \ldots, B_{n}\right)$ be $n$-tuples of operators in $B(H)$. We define the elementary operator $E_{A, B}: B(H) \mapsto B(H)$ by $E_{A, B}(X)=\sum_{i=1}^{n} A_{i} X B_{i}-X$.

Denote $E_{A, A}=E_{A}$. In this section, we prove that if $\sum_{i=1}^{n} A_{i} A_{i}^{*} \leq 1, \sum_{i=1}^{n} A_{i}^{*} A_{i}$ $\leq 1, \sum_{i=1}^{n} B_{i} B_{i}^{*} \leq 1, \sum_{i=1}^{n} B_{i}^{*} B_{i} \leq 1, \operatorname{ker} E_{A, B} \subseteq \operatorname{ker} E_{A^{*}, B^{*}}$, and $T \in \operatorname{ker} \Delta_{A, B} \cap C_{p}$, then the map $F_{p}$ defined by $F_{p}(X)=\left\|T-E_{A, B}(X)\right\|_{p}^{p}$ has a global minimizer at $V$ if, and for $1<p<\infty$ only if, $\sum_{i=1}^{n} A_{i} V B_{i}-V=0$. In other words, we have

$$
\left\|T-E_{A, B}(X)\right\|_{p}^{p} \geq\|T\|_{p}^{p}
$$

if, and for $1<p<\infty$ only if, $\sum_{i=1}^{n} A_{i} V B_{i}-V=$. Additionally, we show that if $\operatorname{ker} E_{A, B} \subseteq E_{A^{*}, B^{*}}$ and $T \in \operatorname{ker} E_{A, B} \cap C_{p}(1<p<\infty)$, then the map $F_{p}$ has a critical point at $W$ if and only if $\sum_{i=1}^{n} A_{i} W B_{i}-W=0$, that is, if $D_{W} F_{p}$ is the Frechet derivative of $F_{p}$ at $W$, the set

$$
\left\{W \in L(H): D_{W} F_{p}=0\right\}
$$

coincides with $\operatorname{ker} E_{A, B}$ (the kernel of $E_{A, B}$ ).

Definition 7.1. Let $u(A, B)=\left\{X \in B(H):\left(\sum_{i=1}^{n} C_{i} X C_{i}-X\right) \in C_{p}\right\}$ and let $F_{p}: U \mapsto R^{+}$be the map defined by $F_{p}(X)=\left\|T-\left(\sum_{i=1}^{n} C_{i} X C_{i}-X\right)\right\|_{p}^{p}$, where $T \in \operatorname{ker} E_{C} \cap C_{p}(1 \leq p<\infty)$.

LEMmA 7.2. Let $C=\left(C_{1}, C_{2}, \ldots, C_{n}\right)$ be $n$-tuple of operators in $B(H)$ such that $\sum_{i=1}^{n} C_{i} C_{i}^{*} \leq 1, \sum_{i=1}^{n} C_{i}^{*} C_{i} \leq 1$, and $\operatorname{ker} E_{c} \subseteq \operatorname{ker} E_{c}^{*}$. If $\sum_{i=1}^{n} C_{i}|S|^{p-1} U^{*} C_{i}=$ $|S|^{p-1} U^{*}$, where $p>1$ and $S=U|S|$ is the polar decomposition of $S$, then $\sum_{i=1}^{n} C_{i}|S| U^{*} C_{i}=|S| U^{*}$.

Proof. By the same arguments as in the proof of Lemma 4.3, the proof can be completed.

THEOREM 7.3. Let $C=\left(C_{1}, C_{2}, \ldots, C_{n}\right)$ be $n$-tuple of operators in $B(H)$. If $\sum_{i=1}^{n} C_{i} C_{i}^{*} \leq 1, \sum_{i=1}^{n} C_{i}^{*} C_{i} \leq 1, \operatorname{ker} E_{C} \subseteq \operatorname{ker} E_{C^{*}}$, and $T \in \operatorname{ker} \Delta_{A, B} \cap C_{p}$, then, for $1 \leq p<\infty$, the map $F_{p}$ has a global minimizer at $W$ if, and for $1<p<\infty$ only if, $\sum_{i=1}^{n} C_{i} W C_{i}-W=0$.

Proof. If $\sum_{i=1}^{n} C_{i} C_{i}^{*} \leq 1, \sum_{i=1}^{n} C_{i}^{*} C_{i} \leq 1$, and $\operatorname{ker} E_{c} \subseteq \operatorname{ker} E_{c}^{*}$, it follows from Theorem 4.4 that

$$
\left\|T-\left(\sum_{i=1}^{n} C_{i} X C_{i}-X\right)\right\|_{p}^{p} \geq\|T\|_{p}^{p},
$$

that is, $F_{p}(X) \geq F_{p}(W)$. Conversely, if $F_{p}$ has a minimum, then

$$
\left\|T-\left(\sum_{i=1}^{n} C_{i} W C_{i}-W\right)\right\|_{p}^{p}=\|T\|_{p}^{p} .
$$


Since $U$ is convex, then the set

$$
\mathscr{V}=\left\{T-\left(\sum_{i=1}^{n} C_{i} X C_{i}-X\right) ; X \in \mathcal{U}\right\}
$$

is also convex. Thus Theorem 6.2 implies that $T-\left(\sum_{i=1}^{n} C_{i} W C_{i}-W\right)=T$.

THEOREM 7.4. Let $C=\left(C_{1}, C_{2}, \ldots, C_{n}\right)$ be $n$-tuple of operators in $B(H)$. If $\sum_{i=1}^{n} C_{i} C_{i}^{*} \leq 1, \sum_{i=1}^{n} C_{i}^{*} C_{i} \leq 1$, $\operatorname{ker} E_{c} \subseteq \operatorname{ker} E_{c}^{*}$, and $T \in \operatorname{ker} E_{C} \cap C_{p}$, then, for $1 \leq p<\infty$, the map $F_{p}$ has a critical point at $W$ if, and for $1<p<\infty$ only if,

$$
\sum_{i=1}^{n} C_{i} W C_{i}-W=0
$$

Proof. Let $W, S \in U$ and let $\phi$ and $\varphi$ be two maps defined, respectively, by

$$
\phi: X \longmapsto S-\left(\sum_{i=1}^{n} C_{i} X C_{i}-X\right), \quad \varphi: X \longmapsto\|X\|_{p}^{p} .
$$

Since the Frechet derivative of $F_{p}$ is given by

$$
\mathscr{D}_{W} F_{p}(T)=\lim _{h \rightarrow 0} \frac{F_{p}(W+h T)-F_{p}(W)}{h},
$$

it follows that

$$
\mathscr{D}_{W} F_{p}(T)=\left[\mathscr{D}_{S-\left(\sum_{i=1}^{n} C_{i} W C_{i}-W\right)}\right]\left(\sum_{i=1}^{n} C_{i} T C_{i}-T\right) .
$$

If $W$ is a critical point of $F_{p}$, then $\mathscr{D}_{W} F_{p}(T)=0$ for all $T \in \mathcal{U}$. By applying Theorem 6.1, we get

$$
\begin{aligned}
\mathscr{D}_{W} F_{p}(T) & =p \operatorname{Retr}\left[\left|S-\left(\sum_{i=1}^{n} C_{i} W C_{i}-W\right)\right|^{p-1} W^{*}\left(\sum_{i=1}^{n} C_{i} T C_{i}-T\right)\right] \\
& =p \operatorname{Retr}\left[Y\left(\sum_{i=1}^{n} C_{i} T C_{i}-T\right)\right]=0,
\end{aligned}
$$

where

$$
S-\left(\sum_{i=1}^{n} C_{i} W C_{i}-W\right)=W\left|S-\left(\sum_{i=1}^{n} C_{i} W C_{i}-W\right)\right|
$$

is the polar decomposition of the operator $S-\left(\sum_{i=1}^{n} C_{i} W C_{i}-W\right)$, and

$$
Y=\left|S-\left(\sum_{i=1}^{n} C_{i} W C_{i}-W\right)\right|^{p-1} W^{*} .
$$


An easy calculation shows that

$$
\left(\sum_{i=1}^{n} C_{i} Y C_{i}-Y\right)=0
$$

that is,

$$
\sum_{i=1}^{n} C_{i}\left|S-\left(\sum_{i=1}^{n} C_{i} W C_{i}-W\right)\right|^{p-1} W^{*} C_{i}=|S-(A W B-W)|^{p-1} W^{*} .
$$

It follows from Lemma 7.2 that

$$
\sum_{i=1}^{n} C_{i}\left|S-\left(\sum_{i=1}^{n} C_{i} W C_{i}-W\right)\right| W^{*} C_{i}=\left|S-\left(\sum_{i=1}^{n} C_{i} W C_{i}-W\right)\right| W^{*} .
$$

By taking adjoints, and since $\operatorname{ker} E_{C} \subseteq \operatorname{ker} E_{C^{*}}$, we get

$$
\sum_{i=1}^{n} C_{i}\left(T-\left(\sum_{i=1}^{n} C_{i} W C_{i}-W\right)\right) C_{i}=\left(T-\left(\sum_{i=1}^{n} C_{i} W C_{i}-W\right)\right)
$$

and then

$$
\sum_{i=1}^{n} C_{i}\left[\left(\sum_{i=1}^{n} C_{i} W C_{i}-W\right)\right] C_{i}=\left(\sum_{i=1}^{n} C_{i} W C_{i}-W\right)
$$

Hence

$$
\sum_{i=1}^{n} C_{i} W C_{i}-W \in R\left(E_{C}\right) \cap \operatorname{ker} E_{C^{*}}
$$

It is easy to see that (arguing as in the proof of [14, Proposition 4.3]) if $C=\left(C_{1}, C_{2}, \ldots, C_{n}\right)$ is $n$-tuple of operator in $B(H)$ such that

$$
\sum_{i=1}^{n} C_{i} C_{i}^{*} \leq 1, \quad \sum_{i=1}^{n} C_{i}^{*} C_{i} \leq 1,
$$

$\operatorname{ker} E_{c} \subseteq \operatorname{ker} E_{c}^{*}$, and $T \in \operatorname{ker} \Delta_{C}$, where $T \in B(H)$, then

$$
\left\|T-\Delta_{C} X\right\| \geq\|T\|
$$

holds for all $X \in B(H)$ and for all $T \in \operatorname{ker} E_{c}$. Hence $\sum_{i=1}^{n} C_{i} W C_{i}-W=0$.

Conversely, if $\sum_{i=1}^{n} C_{i} W C_{i}=W$, then $W$ is minimum, and since $F_{p}$ is differentiable, $W$ is a critical point.

THEOREM 7.5. Let $C=\left(C_{1}, C_{2}, \ldots, C_{n}\right)$ be $n$-tuple of operators in $B(H)$. If

$$
\sum_{i=1}^{n} C_{i} C_{i}^{*} \leq 1, \quad \sum_{i=1}^{n} C_{i}^{*} C_{i} \leq 1,
$$


such that $\operatorname{ker} E_{c} \subseteq \operatorname{ker} E_{c}^{*}, S \in \operatorname{ker} E_{C} \cap C_{p}(0<p \leq 1), \operatorname{dim} H<\infty$, and $S-$ $\left(\sum_{i=1}^{n} C_{i} W C_{i}-W\right)$ is invertible, then $F_{p}$ has a critical point at $W$ if $\sum_{i=1}^{n} C_{i} W C_{i}-$ $W=0$.

Proof. Suppose that $\operatorname{dim} H<\infty$. If $\sum_{i=1}^{n} C_{i} W C_{i}-W=0$, then $S$ is invertible by hypothesis. Also $|S|$ is invertible, hence $|S|^{p-1}$ exists for $0<p \leq 1$ taking $Y=|S|^{p-1} U^{*}$, where $S=U|S|$ is the polar decomposition of $S$.

It is known that if

$$
\sum_{i=1}^{n} C_{i} C_{i}^{*} \leq 1, \quad \sum_{i=1}^{n} C_{i}^{*} C_{i} \leq 1, \quad \operatorname{ker} E_{c} \subseteq \operatorname{ker} E_{c}^{*},
$$

the eigenspaces corresponding to distinct nonzero eigenvalues of the compact positive operator $|S|^{2}$ reduce each $C_{i}$ (see [5, Theorem 8] and [14, Lemma 2.3]). In particular, $|S|$ commutes with $C_{i}$ for all $1 \leq i \leq n$. Hence

$$
C_{i}|S|=|S| C_{i}
$$

Since $\sum_{i=1}^{n} C_{i} S^{*} C_{i}=S^{*}$, that is,

$$
\sum_{i=1}^{n} C_{i}|S| U^{*} C_{i}=|S| U^{*}
$$

then

$$
|S|\left(\sum_{i=1}^{n} C_{i} U^{*} C_{i}-U^{*}\right)=0
$$

and since

$$
A|S|^{p-1}=|S|^{p-1} A,
$$

then

$$
\sum_{i=1}^{n} C_{i} Y C_{i}-Y=\sum_{i=1}^{n} C_{i}|S|^{p-1} U^{*} C_{i}-|S|^{p-1} U^{*}=|S|^{p-1}\left(\sum_{i=1}^{n} C_{i} U^{*} C_{i}-U^{*}\right)
$$

so that $\sum_{i=1}^{n} C_{i} Y C_{i}-Y=0$ and $\operatorname{tr}\left[\left(\sum_{i=1}^{n} C_{i} Y C_{i}-Y\right) T\right]=0$ for all $T \in B(H)$. Since

$$
S=S-\left(\sum_{i=1}^{n} C_{i} W C_{i}-W\right)
$$


then

$$
\begin{aligned}
0 & =\operatorname{tr}\left[Y \sum_{i=1}^{n} C_{i} T C_{i}-Y T\right]=\operatorname{tr}\left[Y\left(\sum_{i=1}^{n} C_{i} T C_{i}-T\right)\right] \\
& =p \operatorname{Retr}\left[Y\left(\sum_{i=1}^{n} C_{i} T C_{i}-T\right)\right]=p \operatorname{Retr}\left[|S|^{p-1} U^{*}\left(\sum_{i=1}^{n} C_{i} T C_{i}-T\right)\right] \\
& =\left(\mathscr{D}_{T} \phi\right)\left(\sum_{i=1}^{n} C_{i} T C_{i}-T\right)=\left(\mathscr{D}_{W} F_{p}\right)(T) .
\end{aligned}
$$

THEOREM 7.6. Let $A=\left(A_{1}, A_{2}, \ldots, A_{n}\right)$ and $B=\left(B_{1}, B_{2}, \ldots, B_{n}\right)$ be $n$-tuples of operators in $B(H)$ such that

$$
\sum_{i=1}^{n} A_{i} A_{i}^{*} \leq 1, \quad \sum_{i=1}^{n} A_{i}^{*} A_{i} \leq 1, \quad \sum_{i=1}^{n} B_{i} B_{i}^{*} \leq 1, \quad \sum_{i=1}^{n} B_{i}^{*} B_{i} \leq 1 .
$$

If

$$
\operatorname{ker} E_{A, B} \subseteq \operatorname{ker} E_{A^{*}, B^{*}}
$$

and $T \in \operatorname{ker} E_{A, B} \cap C_{p}$, then for $1 \leq p<\infty$,

(i) the map $F_{p}$ has a global minimizer at $W$ if, and for $1<p<\infty$ only if,

$$
\sum_{i=1}^{n} A_{i} W B_{i}-W=0
$$

(ii) the map $F_{p}$ has a critical point at $W$ if, and for $1<p<\infty$ only if,

$$
\sum_{i=1}^{n} A_{i} W B_{i}-W=0
$$

(iii) for $0<p \leq 1, \operatorname{dim} H<\infty$, and $S-\left(\sum_{i=1}^{n} C_{i} W C_{i}-W\right)$ invertible, $F_{p}$ has a critical point at $W$ if

$$
\sum_{i=1}^{n} A_{i} W B_{i}-W=0
$$

Proof. It suffices to take the Hilbert space $H \oplus H$ and operators (4.10) and apply Theorems 7.3, 7.4, and 7.5.

REMARK 7.7. (1) In Theorem 7.4 , the implication " $W$ is a critical point $\Rightarrow$ $\sum_{i=1}^{n} A_{i} W B_{i}-W=0$ " does not hold in the case $0<p \leq 1$ because the functional calculus argument involving the function $t \mapsto t^{1 /(p-1)}$, where $0 \leq t<\infty$, is only valid for $1<p<\infty$.

(2) The set $S=\left\{X: A X B-X \in C_{p}\right\}$ contains $C_{p}$. If $X \in C_{p}$, then $X \in S$ and, for example, $I \in S$ but $I \notin C_{p}$. If $A \in C_{p}$, the conclusion of Theorem 7.6 holds for all $X \in B(H)$. 
ACKNOWLEDGment. This work was supported by the Research Center Project No. Math/1422/10.

\section{REFERENCES}

[1] J. H. Anderson, On normal derivations, Proc. Amer. Math. Soc. 38 (1973), 135140.

[2] S. Bouali and S. Cherki, Approximation by generalized commutators, Acta Sci. Math. (Szeged) 63 (1997), no. 1-2, 273-278.

[3] R. G. Douglas, On the operator equation $S^{*} X T=X$ and related topics, Acta Sci. Math. (Szeged) 30 (1969), 19-32.

[4] B. P. Duggal, A remark on normal derivations, Proc. Amer. Math. Soc. 126 (1998), no. 7, 2047-2052.

[5] I. C. Gohberg and M. G. Krein, Introduction to the Theory of Linear Nonselfadjoint Operators, Translations of Mathematical Monographs, vol. 18, American Mathematical Society, Rhode Island, 1969.

[6] D. Kečkić, Orthogonality in $C_{1}$ and $C$-spaces and normal derivations, to appear in J. Operator Theory.

[7] F. Kittaneh, Operators that are orthogonal to the range of a derivation, J. Math. Anal. Appl. 203 (1996), no. 3, 868-873.

[8] F. Kittaneh and R. Younis, Smooth points of certain operator spaces, Integral Equations Operator Theory 13 (1990), no. 6, 849-855.

[9] P. J. Maher, Commutator approximants, Proc. Amer. Math. Soc. 115 (1992), no. 4, 995-1000.

[10] S. Mecheri, On minimizing $\|S-(A X-X B)\|_{p}^{p}$, Serdica Math. J. 26 (2000), no. 2, 119-126.

[11] On orthogonality in von Neumann-Schatten class, Int. J. Appl. Math. 8 (2002), no. 4, 441-447.

[12] S. Mecheri and M. Bounkhel, Some variants of Anderson's inequality in $C_{1}$-classes, JIPAM. J. Inequal. Pure Appl. Math. 4 (2003), no. 1, Article 24, 1-6.

[13] R. Schatten, Norm Ideals of Completely Continuous Operators, Ergebnisse der Mathematik und ihrer Grenzgebiete. N. F., vol. 27, Springer-Verlag, Berlin, 1960.

[14] Y. S. Tong, Kernels of generalized derivations, Acta Sci. Math. (Szeged) 54 (1990), no. 1-2, 159-169.

[15] T. Yoshino, Remark on the generalized Putnam-Fuglede theorem, Proc. Amer. Math. Soc. 95 (1985), no. 4, 571-572.

Salah Mecheri: Department of Mathematics, College of Science, King Saud University, P.O. Box 2455, Riyadh 11451, Saudi Arabia

E-mail address: mecheri@ksu.edu.sa 


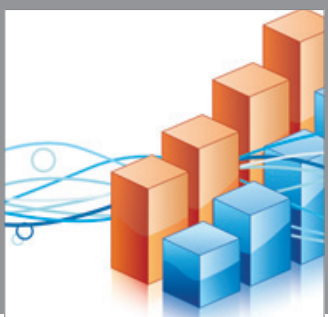

Advances in

Operations Research

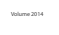

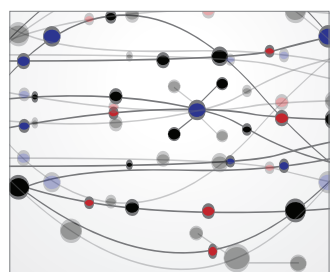

\section{The Scientific} World Journal
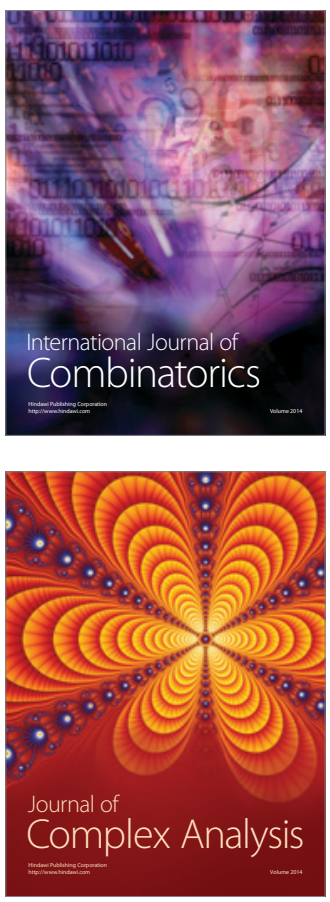

International Journal of

Mathematics and

Mathematical

Sciences
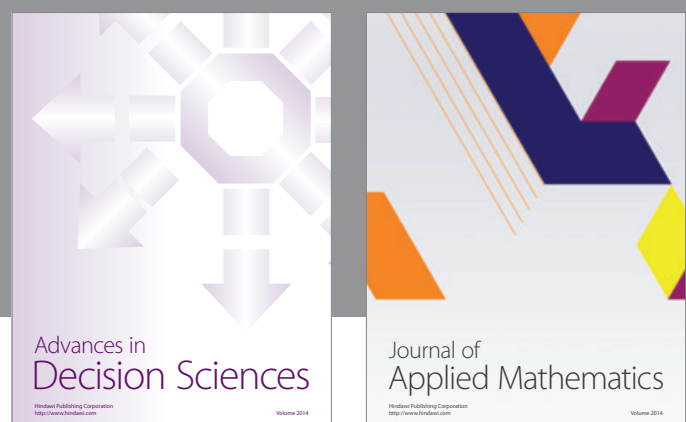

Journal of

Applied Mathematics
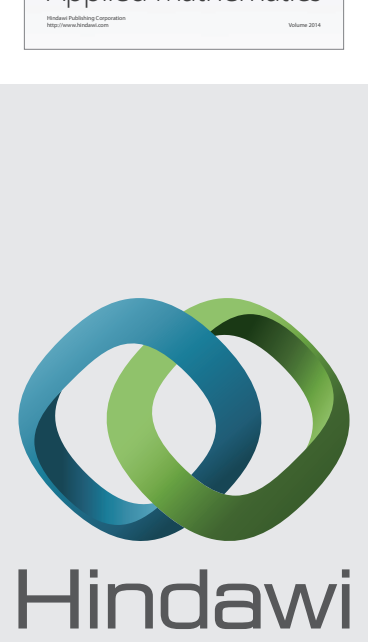

Submit your manuscripts at http://www.hindawi.com
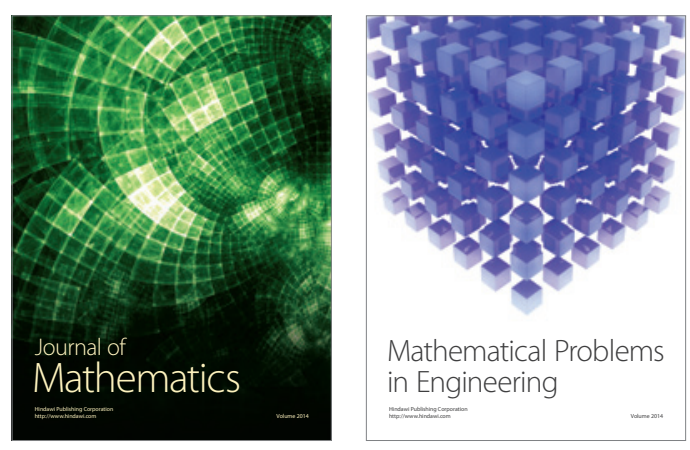

Mathematical Problems in Engineering
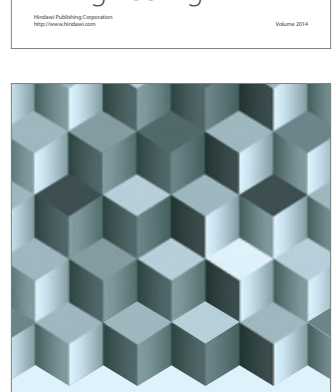

Journal of

Function Spaces
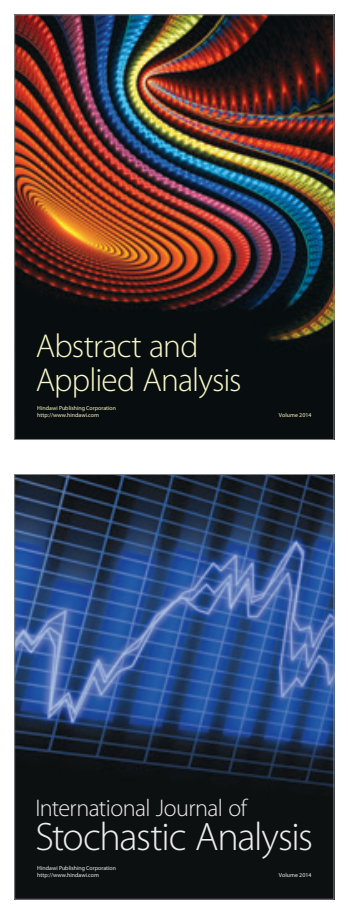

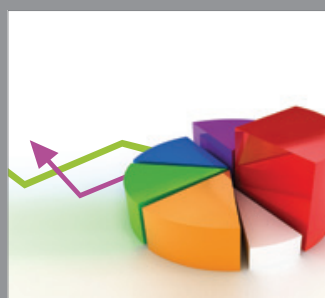

ournal of

Probability and Statistics

Promensencen
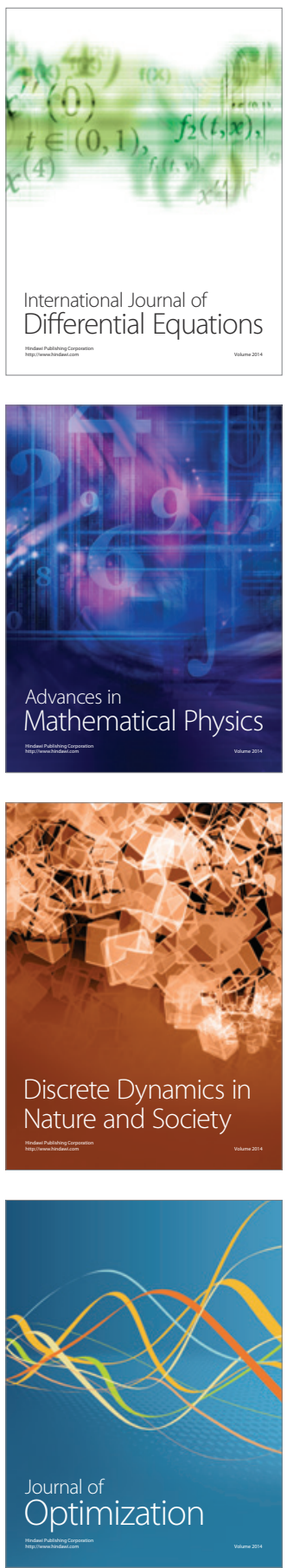